\title{
Intracranial Blood Flow Velocity in Patients with $\beta$-Thalassemia Intermedia Using Transcranial Doppler Sonography: A Case-Control Study
}

\author{
Nahid Ashjazadeh, ${ }^{1}$ Sajad Emami, ${ }^{1}$ Peyman Petramfar, ${ }^{1}$ Ehsan Yaghoubi, ${ }^{1}$ \\ and Mehran Karimi \\ ${ }^{1}$ Shiraz Neuroscience Research Center, Department of Neurology, Shiraz University of Medical Sciences, Shiraz, Iran \\ ${ }^{2}$ Hematology Research Center, Shiraz University of Medical Sciences, Shiraz, Iran \\ Correspondence should be addressed to Mehran Karimi, karimim@sums.ac.ir \\ Received 4 September 2011; Accepted 1 November 2011 \\ Academic Editor: Sezaneh Haghpanah
}

Copyright @ 2012 Nahid Ashjazadeh et al. This is an open access article distributed under the Creative Commons Attribution License, which permits unrestricted use, distribution, and reproduction in any medium, provided the original work is properly cited.

\begin{abstract}
Introduction. Patients with $\beta$-thalassemia intermedia have a higher incidence of thromboembolic events compared to the general population. Previous studies have shown that patients with sickle cell disease, who are also prone to ischemic events, have higher intracranial arterial blood flow velocities measured by transcranial Doppler sonography (TCD). The aim of this study is to evaluate intracranial arterial flow velocities in patients with $\beta$-thalassemia intermedia and compare the results with those found in healthy subjects. Methods. Sixty-four patients with $\beta$-thalassemia intermedia and 30 healthy subjects underwent transcranial Doppler sonography. Results. Significantly higher flow velocities were found in intracranial arteries of patients compared to controls $(P=$ 0.001). Previously splenectomized patients with thrombocytosis showed higher flow velocities than nonsplenectomized patients without thrombosis. Conclusion. The increased flow velocities in patients with $\beta$-thalassemia intermedia may point to a higher risk of ischemic events. Preventive measures such as blood transfusion or antiplatelet treatment may be beneficial in these patients.
\end{abstract}

\section{Introduction}

Patients with $\beta$-thalassemia intermedia (B-TI) seem to show higher rates of thromboembolic events than individuals without thalassemia or patients with $\beta$-thalassemia major, in particular if they have been splenectomized [1]. It is estimated that $4 \%$ of patients with $\beta$-thalassemia intermedia will experience a thromboembolic event [2]. Previous splenectomy and thrombocytosis and/or platelet abnormalities are major factors associated with thromboembolic events in patients with $\beta$-thalassemia intermedia $[1,3,4]$, and ischemic stroke is increasingly recognized as one of the most devastating complications of this disease [5].

Ischemic stroke is also a known complication of sickle cell disease [6]. In a prospective study in patients with this condition, higher blood flow velocity in the intracranial arteries was associated with a higher risk of ischemic stroke [7]. The stroke prevention trial in sickle cell anemia (STOP) has indicated a role for transcranial Doppler sonography
(TCD) in measuring intracranial arterial flow velocities to identify sickle cell patients at a high risk of ischemic stroke [8]. TCD measurement of intracranial flow velocities is of aid in deciding when to start blood transfusion in these patients to reduce the risk of ischemic stroke $[8,9]$.

An association between TCD findings and stroke risk has been confirmed in sickle cell disease; however, as far as we know, no studies have been conducted to evaluate TCD findings in patients with $\beta$-thalassemia intermedia, another high-risk group for ischemic stroke. In the present study, we compared the intracranial arterial flow velocities of $\beta$-thalassemia intermedia patients with those of healthy subjects.

\section{Patients and Methods}

This is a case-control study conducted in a tertiary outpatient clinic affiliated with Shiraz University of Medical Sciences, Southern Iran, for a period of one year during 2009. 
Consecutive patients older than 15 years of age with confirmed $\beta$-thalassemia intermedia by complete blood count and hemoglobin electrophoresis who were referred to an outpatient thalassemia clinic enrolled in the study. Diagnosis of B-TI was based on complete blood count, hemoglobin electrophoresis, and initial hemoglobin $(\mathrm{Hb})$ level of $7 \mathrm{gr} / \mathrm{dL}$, and age of diagnosed anemia was after 2. All of them were transfusion independent. Patients were recruited at a routine follow-up visit with a hematologist in the clinic. Exclusion criteria were a history of diabetes mellitus, hypertension, ischemic heart disease, thrombosis, previous cerebrovascular disease, sickle cell anemia, or inadequate temporal window for TCD. The study was approved by the Ethics Committee of Shiraz University of Medical Sciences (no. 2885), and written informed consent to participate was obtained from all patients or their first-degree families. All patients were receiving folic acid ( $5 \mathrm{mg} /$ day) and hydroxyurea (8$15 \mathrm{mg} / \mathrm{kg} /$ day).

For each patient, we completed a data collection form that included age, sex, place of residence, prior splenectomy, prior transfusion, history of thrombosis, previous stroke or transient ischemic attack, and laboratory information (complete blood cell count, ferritin, blood urea nitrogen, creatinine, alanine aminotransferase, aspartate aminotransferase, alkaline phosphatase, and albumin). Thrombocytosis was defined as a platelet count $>500,000 / \mathrm{dL}$.

Transcranial Doppler ultrasound was carried out in all patients. All TCD studies were performed by one investigator using a Legend TC22 transcranial Doppler ultrasound unit (Bristol, UK) with a 2-MHz transducer. The two middle cerebral arteries (MCAs), anterior cerebral arteries (ACAs), posterior cerebral arteries (PCAs), and the terminal internal carotid arteries were insonated using a temporal window approach (Eleven patients had poor temporal window who were excluded from the study). The basilar artery (BA) and vertebral arteries were examined through a suboccipital approach in sitting position. The highest mean flow velocity of each artery was recorded separately.

The control group consisted of 30 sex/age-matched subjects with no known hematologic disease. The same exclusion criteria as for the patients were used for the controls. All the control subjects underwent TCD examination with the same protocol.

The Mann-Whitney $U$ test, Pearson correlation coefficient $(r)$, and $t$-test were used for comparison of the variables between groups. Results are expressed as percentages and absolute frequencies, where appropriate. Descriptive results are presented as the mean \pm standard deviation (SD). $P$ values $<0.05$ were considered significant. Statistical analyses were performed in SPSS version 15.0 (SPSS, Chicago, Ill, USA).

\section{Results}

After applying the inclusion and exclusion criteria, 64 patients with $\beta$-thalassemia intermedia and 30 healthy subjects were recruited. There were no significant differences between patients and controls according to sex (male: $40.6 \%$
TABLE 1: Mean intracranial arterial flow velocities in $\beta$-thalassemia intermedia patients with or without splenectomy.

\begin{tabular}{lccc}
\hline & $\begin{array}{c}\text { Without } \\
\text { splenectomy }\end{array}$ & $\begin{array}{c}\text { With } \\
\text { splenectomy }\end{array}$ & $P$ value \\
\hline Left ICA & $84.6 \pm 24.4$ & $94.1 \pm 20.7$ & 0.097 \\
Right ICA & $83.1 \pm 25.6$ & $86.5 \pm 25.1$ & 0.538 \\
Left MCA & $66.6 \pm 26.9$ & $87.9 \pm 23.9$ & 0.001 \\
Right MCA & $71.3 \pm 28.2$ & $85.8 \pm 22.7$ & 0.026 \\
Left ACA & $58.2 \pm 19.7$ & $73.4 \pm 18.3$ & 0.002 \\
Right ACA & $57.3 \pm 19.0$ & $69.2 \pm 18.2$ & 0.013 \\
Left PCA & $35.1 \pm 11.7$ & $42.1 \pm 11.6$ & 0.021 \\
Right PCA & $34.8 \pm 10.8$ & $41.7 \pm 14.3$ & 0.037 \\
Left vertebral & $52.2 \pm 15.0$ & $58.3 \pm 14.5$ & 0.102 \\
artery & & & \\
Right vertebral & $55.6 \pm 15.3$ & $62.4 \pm 17.5$ & 0.106 \\
artery & $66.1 \pm 17.2$ & $76.7 \pm 16.6$ & 0.016 \\
Basilar artery &
\end{tabular}

ICA, internal carotid artery; MCA, middle cerebral artery; ACA, anterior cerebral artery; PCA, posterior cerebral artery.

versus $53.3 \% ; P=0.251)$ or age $(23.6 \pm 5.2$ versus $25.4 \pm 5.4$; $P=0.001)$. Mean velocities of all mentioned vessels were measured in all patients and control subjects, and no missing vessel was detected. Among the patients, 54.7\% (35/64) had undergone splenectomy, and $9.4 \%(6 / 64)$ had received a blood transfusion once or twice a year before the study. None of the control subjects had received transfusion. In patients, mean $\mathrm{Hb}$ level was $9.3 \pm 1.2 \mathrm{~g} / \mathrm{dL}$ (range 6.9-12.3), mean white blood cell count was $8873 \pm 2004 /$ dL (range 4900 13800 ), and mean platelet count was $523 \times 10^{3} \pm 219 \times 10^{3} / \mathrm{dL}$ (range $188 \times 10^{3}-1035 \times 10^{3}$ ). There were no significant differences in age, white blood cell count, or Hb level between splenectomized and nonsplenectomized patients $(P>0.05)$. Mean platelet count was significantly higher in patients who had undergone splenectomy $\left(696 \times 10^{3} \pm 129 \times 10^{3} / \mathrm{dL}\right.$ versus $\left.315 \times 10^{3} \pm 78 \times 10^{3} / \mathrm{dL} ; P=0.001\right)$. All the splenectomized patients had thrombocytosis, and none of the nonsplenectomized patients had thrombocytosis. Platelet count correlated with blood flow velocity in the right MCA $(r=0.291, P=0.020)$, left MCA $(r=0.366, P=0.003)$, left ACA $(r=0.258, P=0.040)$, right PCA $(r=0.270$, $P=0.031)$, left PCA $(r=0.267, P=0.033)$, and BA $(r=0.300, P=0.016)$. There were no correlations between platelet count and blood flow velocities of the other arteries $(P>0.05)$. Mean intracranial arterial flow velocities in $\beta$ thalassemia intermedia patients with or without splenectomy are shown in Table 1. There were no significant differences in white blood cell count, Hb levels, or platelet count between patients who had undergone transfusion and those who had not $(P>0.05)$.

Comparison of the mean intracranial artery flow velocities between patients and controls showed significantly higher velocities in all intracranial arteries of patients $(P=$ $0.001)$. Mean flow velocities recorded in patients and controls are shown in Table 2. 
TABle 2: Mean intracranial arterial flow velocities in $\beta$-thalassemia intermedia patients and healthy controls.

\begin{tabular}{lccc}
\hline & Patients & Healthy subjects & $P$ value \\
\hline Left ICA & $89.8 \pm 22.8$ & $52.1 \pm 8.6$ & 0.001 \\
Right ICA & $84.9 \pm 21.7$ & $50.0 \pm 8.4$ & 0.001 \\
Left MCA & $78.3 \pm 27.3$ & $49.4 \pm 9.1$ & 0.001 \\
Right MCA & $79.3 \pm 26.2$ & $48.7 \pm 7.6$ & 0.001 \\
Left ACA & $66.5 \pm 20.3$ & $44.4 \pm 9.5$ & 0.001 \\
Right ACA & $63.8 \pm 19.4$ & $42.5 \pm 7.8$ & 0.001 \\
Left PCA & $38.9 \pm 12.1$ & $29.7 \pm 6.6$ & 0.001 \\
Right PCA & $38.6 \pm 13.2$ & $27.3 \pm 6.3$ & 0.001 \\
Left vertebral & $55.5 \pm 14.9$ & $29.5 \pm 4.6$ & 0.001 \\
artery & & & \\
Right vertebral & $59.3 \pm 16.8$ & $29.3 \pm 4.6$ & 0.001 \\
artery & $71.9 \pm 17.6$ & $47.1 \pm 8.2$ & 0.001 \\
Basilar artery &
\end{tabular}

ICA, internal carotid artery; MCA, middle cerebral artery; ACA, anterior cerebral artery; PCA, posterior cerebral artery.

\section{Discussion}

In this study, higher blood flow velocities were found in all intracranial arteries of our patients. Flow velocity was higher in most arteries of splenectomized patients compared to nonsplenectomized patients, especially in the anterior circulation. In addition, there was a correlation between platelet count and flow velocities.

Numerous studies in sickle cell anemia have evaluated the role of TCD as a screening tool to identify and followup patients at high risk of ischemic stroke [7-10]. TCD can also detect asymptomatic cerebrovascular disease in patients with sickle $\beta$-thalassemia [11], and the findings on TCD study show a good correlation with cerebral angiography [12]. However, no such studies have been performed in $\beta$-thalassemia intermedia, another hematologic disease in which there is a substantial associated risk of ischemic stroke. Screening of asymptomatic $\beta$-thalassemia intermedia patients by TCD has not been reported previously, and the impact of this measure on further stroke remains to be defined. TI patients are prone to thromboembolic event, especially those patients associated with splenectonomy and thrombocytosis [2,3].

There is growing evidence that increased intracranial arterial flow velocities associate with a higher risk of ischemic stroke $[7,13]$. The recommendation for chronic red blood cell transfusion in patients with high stroke risk is based on findings from the STOP trial, in which patients with high intracranial arterial flow velocities showed a $90 \%$ reduction in stroke rate following this treatment [8]. Patients with $\beta$ thalassemia intermedia do not regularly receive blood transfusion, and they have a higher risk of ischemic stroke than $\beta$-thalassemia major patients, who are often treated with transfusion [3]. In addition, a higher rate of thromboembolic events occurs in splenectomized $\beta$-thalassemia intermedia patients $[3,14]$, who usually have a higher platelet count.
The presence of a high flow velocity in patients with $\beta$ thalassemia intermedia may herald a cerebrovascular event and indicate a need for special attention. The association of flow velocity with splenectomy and platelet count suggests that these two factors should also be taken into account when interpreting stroke risk in a patient with $\beta$-thalassemia intermedia.

This study has some limitations. Patients were not prospectively followedup to clarify the impact of higher blood flow velocities on the risk of ischemic stroke. The interpretation presented here is based on previous studies, mainly in sickle cell disease, which showed a higher risk of stroke in association with higher flow velocities measured by TCD. Furthermore, we did not measure flow velocities before and after blood transfusion to estimate the impact of this measure on reducing velocities.

In conclusion, in this first study evaluating TCD findings in $\beta$-thalassemia intermedia patients, higher intracranial arterial blood flow velocities were found in comparison to normal subjects. These findings may indicate that these patients are at a higher risk of ischemic events and that preventive measures, such as blood transfusion or antiplatelet drug administration, could be beneficial. Nonetheless, prospective randomized clinical trials would be needed to establish recommendations in this regard.

\section{Conflict of Interests}

All the authors declare that they have no conflict of interests.

\section{Acknowledgments}

This study was financially supported by Shiraz University of Medical Sciences. They thank Shirin Parand at the Hematology Research Center for help with paper preparation, and C. Cavallo (author aid in the Eastern Mediterranean) for editing and improving the use of English in the paper. This paper is relevant to the thesis of S. Emami with Project no. 2885.

\section{References}

[1] A. T. Taher, Z. K. Otrock, I. Uthman, and M. D. Cappellini, "Thalassemia and hypercoagulability," Blood Reviews, vol. 22, no. 5, pp. 283-292, 2008.

[2] A. Taher, H. Isma'eel, G. Mehio et al., "Prevalence of thromboembolic events among 8,860 patients with thalassaemia major and intermedia in the Mediterranean area and Iran," Thrombosis and Haemostasis, vol. 96, no. 4, pp. 488-491, 2006.

[3] M. D. Cappellini, L. Robbiolo, B. M. Bottasso, R. Coppola, G. Fiorelli, and P. M. Mannucci, "Venous thromboembolism and hypercoagulability in splenectomized patients with thalassaemia intermedia," British Journal of Haematology, vol. 111, no. 2, pp. 467-473, 2000.

[4] A. Tripodi, M. D. Cappellini, V. Chantarangkul et al., "Hypercoagulability in splenectomized thalassemic patients detected by whole-blood thromboelastometry, but not by thrombin generation in platelet-poor plasma," Haematologica, vol. 94, no. 11, pp. 1520-1527, 2009. 
[5] M. Karimi, M. Khanlari, and E. A. Rachmilewitz, "Cerebrovascular accident in $\beta$-thalassemia major $(\beta$-TM) and $\beta$ thalassemia intermedia $(\beta$-TI)," American Journal of Hematology, vol. 83, no. 1, pp. 77-79, 2008.

[6] K. Ohene-Frempong, S. J. Weiner, L. A. Sleeper et al., "Cerebrovascular accidents in sickle cell disease: rates and risk factors," Blood, vol. 91, no. 1, pp. 288-294, 1998.

[7] R. J. Adams, V. C. McKie, E. M. Carl et al., "Long-term stroke risk in children with sickle cell disease screened with transcranial Doppler," Annals of Neurology, vol. 42, no. 5, pp. 699-704, 1997.

[8] M. T. Lee, S. Piomelli, S. Granger et al., "Stroke prevention trial in sickle cell anemia (STOP): extended follow-up and final results," Blood, vol. 108, no. 3, pp. 847-852, 2006.

[9] N. Venketasubramanian, I. Prohovnik, A. Hurlet, J. P. Mohr, and S. Piomelli, "Middle cerebral artery velocity changes during transfusion in sickle cell anemia," Stroke, vol. 25, no. 11, pp. 2153-2158, 1994.

[10] J. J. Seibert, C. M. Glasier, R. S. Kirby et al., "Transcranial Doppler, MRA, and MRI as a screening examination for cerebrovascular disease in patients with sickle cell anemia: an 8-year study," Pediatric Radiology, vol. 28, no. 3, pp. 138-142, 1998.

[11] D. I. Zafeiriou, M. Prengler, N. Gombakis et al., "Central nervous system abnormalities in asymptomatic young patients with $\mathrm{S} \beta$-thalassemia," Annals of Neurology, vol. 55, no. 6, pp. 835-839, 2004.

[12] R. J. Adams, F. T. Nichols, R. Figueroa, V. McKie, and T. Lott, "Transcranial Doppler correlation with cerebral angiography in sickle cell disease," Stroke, vol. 23, no. 8, pp. 1073-1077, 1992.

[13] R. Adams, V. McKie, F. Nichols et al., "The use of transcranial ultrasonography to predict stroke in sickle cell disease," New England Journal of Medicine, vol. 326, no. 9, pp. 605-610, 1992.

[14] M. Karimi, H. Bagheri, F. Rastgu, and E. A. Rachmilewitz, "Magnetic resonance imaging to determine the incidence of brain ischaemia in patients with $\beta$-thalassaemia intermedia," Thrombosis and Haemostasis, vol. 103, no. 5, pp. 989-993, 2010. 


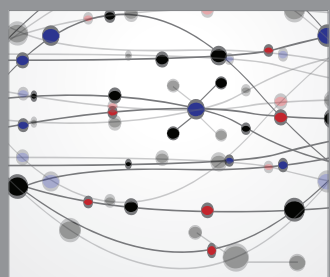

The Scientific World Journal
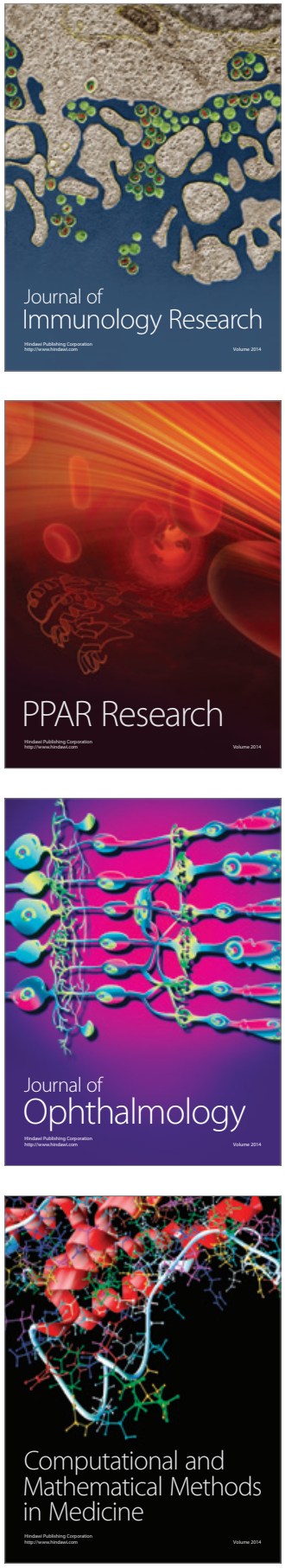

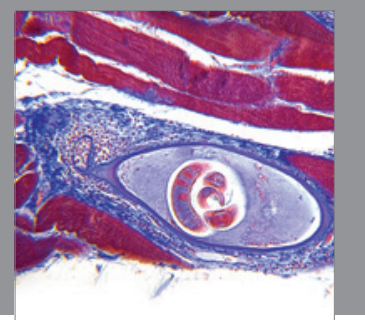

Gastroenterology

Research and Practice
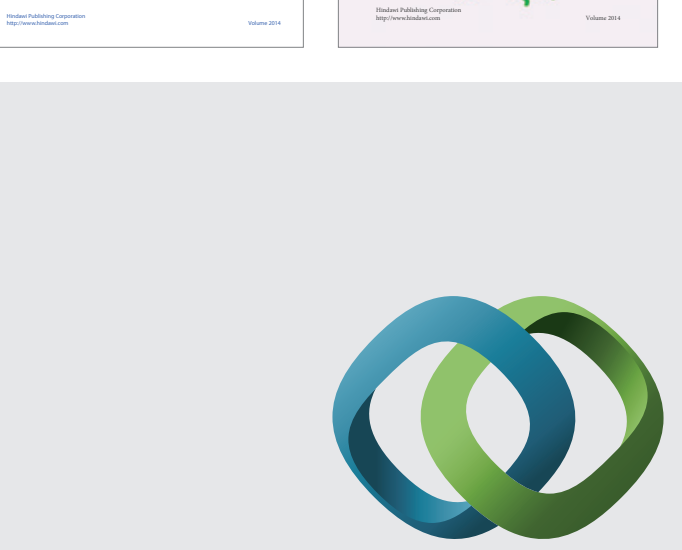

\section{Hindawi}

Submit your manuscripts at

http://www.hindawi.com
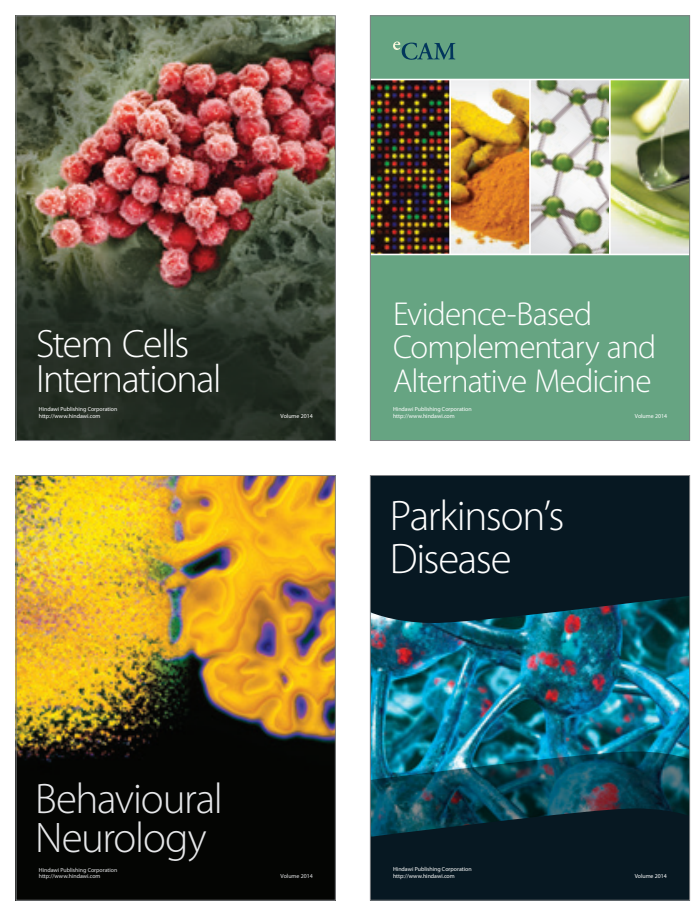

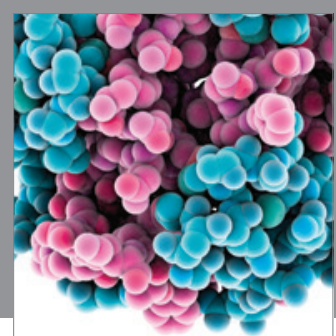

Journal of
Diabetes Research

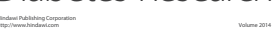

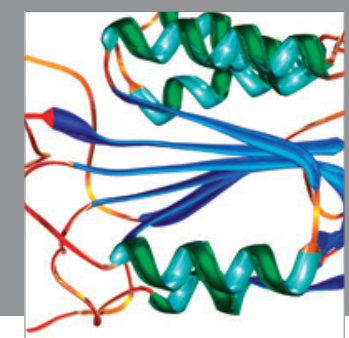

Disease Markers
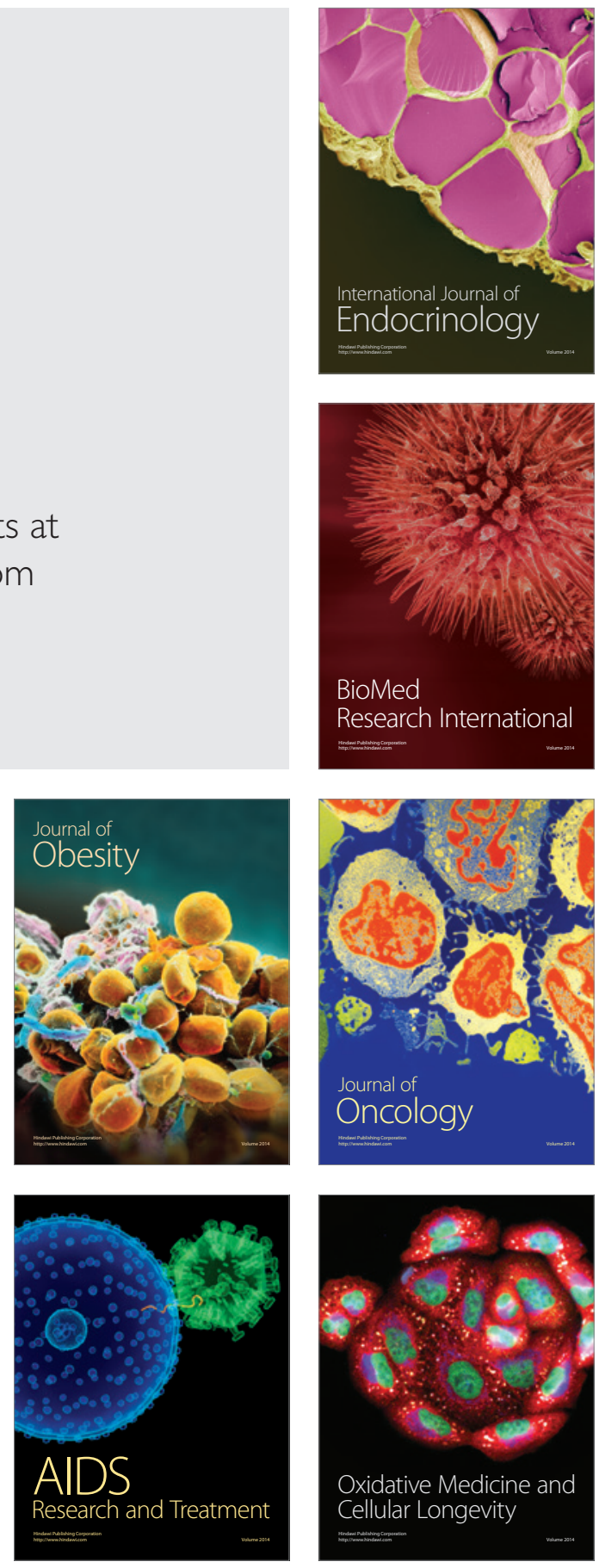